\title{
The retinoprotective role of phenytoin
}

This article was published in the following Dove Press journal:

Drug Design, Development and Therapy

\author{
Silvia Bartollino' \\ Flavia Chiosi' \\ Silvio di Staso ${ }^{2}$ \\ Maurizio Uva ${ }^{3}$ \\ Arduino Pascotto' \\ Michele Rinaldi ${ }^{4}$ \\ Jan M Keppel Hesselink ${ }^{5}$ \\ Ciro Costagliola'
}

'Eye Clinic, Department of Medicine and Health Sciences "V.Tiberio," University of Molise, Campobasso, Italy; ${ }^{2}$ Department of Surgical Science, Ophthalmic Clinic, University of L'Aquila, L'Aquila, Italy; ${ }^{3}$ Eye Clinic, University of Catania, Catania, Italy; ${ }^{4}$ Department of Ophthalmology, University della Campania Luigi Vanvitelli, Naples, Italy; ${ }^{5}$ Department of Health, University of Witten/ Herdecke, Witten, Germany
Correspondence: Flavia Chiosi Department of Medicine and Health Sciences, Università degli Studi del Molise, Via Francesco De Sanctis, I 86100 Campobasso, Molise, Italia Tel +393395333929

Fax +39087440 4886

Email flaviachiosi@yahoo.it

\begin{abstract}
Phenytoin is a non-sedative barbiturate derivate and has been recently rediscovered as a neuroprotective and retinoprotective compound in patients affected by optic neuritis secondary to multiple sclerosis. However, currently there are still no neuroprotective compounds registered and available in the clinic. We reviewed the literature supporting the retinoprotective properties of phenytoin and analyzed the various approaches and definitions from the first research periods onwards. The retinoprotective role of phenytoin was already known in the 1970s, but only recently has this effect been rediscovered, confirming that it could indeed provide structural protection of the retinal cells.
\end{abstract}

Keywords: phenytoin, neuroprotection, retinoprotection, optic neuritis, multiple sclerosis

\section{Introduction}

The concept of neuroprotection can be found in the literature from the 1940s onwards and it was initially based on clinical endpoints such as the protection against convulsions due to anticonvulsants, barbiturates and phenytoin. Since the retinal neurons are nerve cells, neuroprotection played an essential role in maintaining retina structures too. The scientific study of neuroprotection focuses on promising translational pharmacological advances related to the preservation of the structure and function of photoreceptors. Progress has been made to clarify the underlying mechanisms of photoreceptor and ganglion cell degeneration and to identify potential neuroprotective strategies.

There has been growing interest in the use of antiepileptic drugs (AEDs) for neuroprotection along with increasing our understanding of the mechanisms underlying brain injury. However, defining the clinical paradigms and selecting appropriate outcomes to detect neuroprotective effects presented a plethora of challenges to clinicians studying the neuroprotective properties of drugs. Established AEDs, such as phenytoin, phenobarbital and carbamazepine, have shown neuroprotective activity in an ischemic/hypoxic model of neuronal injury. ${ }^{1}$

Neuroprotection has also played a pivotal role in maintaining retina structures. Along with the neuroprotection, the concept of retinoprotection has also evolved tremendously in recent years. The science of neuroprotection has focused on promising translational pharmacological studies related to the preservation of the physiology of photoreceptors. ${ }^{2}$ Progress has been made to clarify the underlying mechanisms of photoreceptor and ganglion cell degeneration and to identify potential neuroprotective strategies. ${ }^{3}$

Phenytoin is a selective sodium-channel inhibitor used as an anticonvulsant in the treatment of epilepsy. It acts as a neuroprotective agent at therapeutic concentrations in experimental models. ${ }^{4}$ It has been documented in animal experiments that phenytoin could reduce mortality resulting from anoxia or hypoxia and the major endpoint 
considered was "survival time." The protective effects of phenytoin have been defined at the cellular, tissue, and organ levels, and the mechanism of action of phenytoin is based on inhibiting the increase of intracellular sodium. Phenytoin can be loaded rapidly to achieve therapeutic serum concentrations within days. ${ }^{5}$ These properties make this compound ideal as a potential neuroprotectant because it has been demonstrated to have neuroprotective properties in the laboratory and animal models, in addition to efficacy in clinical trials. In this chapter, we briefly review the scientific background for the retinoprotective role of phenytoin in neurodegenerative retinal diseases and its potential as a sodium-channel inhibitor in the context of these pathologies.

\section{Phenytoin and optic neuritis}

We searched PubMed, Medline and the Cochrane Database of Systematic Reviews, using the terms "phenytoin" or "sodium channels," "clinical trials" and "neuroprotection." We found several pre-clinical studies, case reports, and randomized controlled trials of neuroprotection in patients with acute optic neuritis.

The first report dates back to 1972 , with a series of seven patients affected by ischemic optic neuritis. ${ }^{6}$ Under phenytoin therapy, four of these patients clearly improved, whereas the other three did not show any improvement and instead developed visual defects in the subsequent years. However, ophthalmologists noticed that vision improvement in patients undergoing phenytoin therapy might have been related to the natural progression of the disease. A controlled double-blind study at that time was in progress, even though the results of this prospective clinical trial were apparently never published.

Even so, in a double-blind study of 15 patients with residual visual impairment after acute ischemic optic neuropathy, neither visual acuity nor visual fields improved during the phenytoin treatment. ${ }^{7}$ To investigate the retinoprotective effects of phenytoin on the electroretinogram variations secondary to hypoxia, an in vitro rabbit retina preparation was conducted. Phenytoin protected the retinal activity from the inhibition of oxygen deprivation. This effect was dependent on the potassium concentrations in the extracellular compartment. ${ }^{8}$ Inexplicably, no further studies were performed despite the promising results that were initially observed in these studies.

Comparable functional read-outs have also been used more often to support the claim that phenytoin had a neuroprotective effect. In an in vitro anoxic optic nerve model, phenytoin showed protective effects, which were measured through electrophysiological parameters (recovery of the Compound Action Potential) at a concentrations of $1 \mu \mathrm{M} .{ }^{9}$ The recovery was around $60 \%$ and the authors pointed out that the protective effect occurred at one fifth of the levels normally measured in the plasma. This concentration is lower than that required to block neuronal sodium channels. At that time, glutamate release during ischemia was also considered a critical factor in eliciting permanent electrical changes caused by a pathological event, and the neuroprotective effect of agents interfering with glutamate release were examined in experimental ischemia models. The study by Calabresi et al ${ }^{10}$ investigated the possible role of ionotropic glutamate receptors and in particular AMPA-like receptors in the expression of irreversible electrophysiological changes induced by in vitro ischemia, in addition to whether the neuroprotective action of various neurotransmitter agonists were related to a presynaptic inhibitory action at glutamatergic synapses. The authors determined that AMPAlike receptors played a role in the irreversible loss of field potential amplitude induced by ischemia in the striatum and they suggested that agents that reduce glutamatergic corticostriatal transmission, might have a neuroprotective effect. However, their efficacy did not seem to be directly related to their capability to decrease glutamate release from corticostriatal terminals. An additional modulatory effect on voltage-dependent conductances and on ischemia-induced ion distribution at the postsynaptic site was also hypothesized to play a crucial role.

While the protective effect of orally administered phenytoin on the optic nerve was described by Lo et al in a model of allergic encephalomyelitis, the main structural endpoint considered was the loss of optic nerve axons. ${ }^{11}$ The plasma levels of phenytoin were brought into the therapeutic range. There was a significant sparing effect: phenytoin reduced the loss of optic nerve axons from $49 \%$ to $12 \%$, while significantly reducing the paralysis. The authors concluded that the treatment with phenytoin had a robust protective effect in experimental allergic encephalomyelitis mice, preserving most axons.

In 2002 Thakral et al $^{12}$ reported an isolated case of phenytoin-induced toxicity in a young patient affected by cryptogenic simple partial and secondary generalized epilepsy. Visual dysfunction was evidenced through flash visually evoked potentials and an electroretinogram, which was partially recovered by reduced serum levels of phenytoin. However clear toxicity-induced phenytoin-related polyneuropathies are extremely rare, and they are always related to high doses or high plasma levels of phenytoin, that primarily develop over many years of therapy. ${ }^{13}$ 
The proof of the concept for neuroprotection and retinoprotection in the clinic emerged in 2016 in the paper by Raftopoulos et al. ${ }^{14}$

Acute optic neuritis (AON) is a common complication of multiple sclerosis (MS), and the attacks can lead to persistent visual impairment through neuroaxonal damage in the retina and optic nerve. Corticosteroids usually hasten recovery, but they do not improve the final outcome. Therefore, to prevent residual disability from relapses, the authors sought a neuroprotective approach. They conducted a randomized clinical trial (RCT), enrolling 86 people with AON, who were randomized within 2 weeks of symptom onset to receive either phenytoin or placebo for 3 months. The primary endpoint was a structural one: retinal nerve fiber layer (RNFL) thickness using optical coherence tomography (OCT) at baseline and at 6 months. However, the researchers pointed out that using the RNFL in the unaffected control eye at baseline for comparison "is prone to error" and in future trials, the use of optical coherence tomography (OCT) segmentation methods would be recommended to avoid this problem. They also suggested that measuring ganglion cells plus inner plexiform layer thickness as an estimate of ganglion neuronal integrity might be a better alternative to RNFL thickness because it does not swell during acute optic neuritis. Treatment with phenytoin led to a significant $(30 \%)$ reduction in the extent of RNFL loss compared to a placebo. The authors concluded that their findings suggested that phenytoin exerts a neuroprotective effect in patients with $\mathrm{AON}$, at concentrations that selectively block voltage-gated sodium channels.

\section{Phenytoin and glaucoma}

Glaucoma is characterized by progressive, irreversible damage to the optic nerve, resulting in serious vision loss and blindness. At present intraocular pressure (IOP) is considered the most important modifiable risk factor for glaucoma onset and progression. Reduction of IOP has been repeatedly demonstrated to be an effective intervention in glaucoma patients, regardless of subtype or disease stage. ${ }^{15}$ However, the direct link between a specific pharmaceutical product to lower IOP and the prevention or delay of visual field progression has not been established nor are these medications specifically approved for such a functional outcome. Even though all approved treatments for glaucoma involve IOP reduction, patients frequently continue to progress despite treatment. Therefore, developing treatments that preserved visual function through mechanisms other than lowering IOP are thought to be beneficial.
During the 1970s, preliminary results revealed the protective effects of phenytoin on retinal degeneration in glaucoma. ${ }^{16}$ The beneficial effects of phenytoin on glaucomatous field loss was reported in a cohort of 17 patients who were administered with phenytoin for a 2-month period. In 11 of these patients the field loss could be stopped or even partly reversed.

Based on the protective effects of phenytoin on glaucomatous visual fields from that period, experimental ophthalmologists further explored the neuroprotective effects of phenytoin in an optic nerve crush model. Here the vasculature remained intact. Ten animals received a single dose of phenytoin (100 mg/ $\mathrm{kg}^{-1}$ body weight), and ten animals were intraperitoneally injected with the vehicle alone 10 minutes prior to the crush injury. ${ }^{17}$ To ascertain the neuroprotective effect, retinal ganglion cell survival was considered the primary endpoint. In these experiments, the ganglion cell survival with and without phenytoin gave the following results: optic nerve crush: $24.0 \pm 4.0$ retinal ganglion cells per high power field. In the crush injury, phenytoin treatment resulted in a larger number of surviving cells: $50.7 \pm 7.3(P<0.001)$. The results for this injury paradigm showed that phenytoin acts as a neuroprotective agent. Secondary endpoints were based on glutamate provocation, supporting this hypothesis for the mechanism of the action of phenytoin. The compound significantly $(P<0.01)$ inhibited the increase in intracellular calcium concentrations after the administration of glutamate Thus, phenytoin clearly counteracts excitotoxic damage.

Subsequently, the evidence supporting the potential retinoprotective and neuroprotective effects of phenytoin encouraged Hains and Waxman ${ }^{18}$ from the Department of Neurology and Center for Neuroscience and Regeneration Research (Yale University School of Medicine, USA) to examine the effects of phenytoin administration in a model for glaucoma. They also chose structural endpoints, and found that phenytoin exerted a neuroprotective effect on retinal ganglion cell (RGC) density, reducing the loss of axons within the optic nerve.

\section{Structural endpoints for retinoprotection}

Because there is consistent evidence in the literature for neuroprotection and retinoprotection as outcome endpoints, we recommend focusing on structural features as primary surrogate endpoints. Moreover, the sensitivity of clinical endpoints is poorer compared to structural endpoints, and we might discard valuable retinoprotective drugs if we focus solely on clinical endpoints. Structural endpoints (such as the 
thickness of the RNFL and OCT segmentations methods used to estimate the ganglion neuronal integrity) might be considered for broad testing, in addition to exploring phenytoin as a retinoprotective drug for use in patients diagnosed with glaucoma and optic neuritis. The authors' research supports the retinoprotective effects of phenytoin, and they associate the use of phenytoin with a significant reduction in the loss of RNFL thickness and macular volume after acute optic neuritis. This emphasizes phenytoin's protective effects in ganglion cells, their axons, and the optic nerve. ${ }^{14}$ Although the results of this study are a major advancement and are undeniably encouraging, future studies need to include more frequent OCT sampling, as well as more detailed OCT segmentation-derived retinal measures. At the average concentrations achieved in this trial, phenytoin was an almost pure activity-dependent inhibitor of voltage-gated sodium channels in an episode of inflammatory demyelination. It might be conceivable that other sodium channel inhibitors could play a role in neuroprotection in patients with acute optic neuritis and in other types of relapse in neurodegenerative disease. This is supported by the similarities between acute optic neuritis and other types of relapse. ${ }^{19}$

Future studies should more precisely establish the optimal therapeutic window for neuroprotection during relapse. What happens in the eye seems to mirror what occurs in the rest of the brain. Thus, this approach could potentially be used to not only treat pathologies, but any flare-ups associated with these diseases. It is reasonable to consider neuroprotective drugs, such as phenytoin, complementary therapies to the existing immunomodulating treatments and potential remyelination approaches.

\section{Future directions}

The evidence presented in this review suggests that there is a protective role of phenytoin in optic neuritis and other cases of relapses for MS. The eye is the most accessible part of the human nervous system, and optic neuritis in MS patients is considered an ideal target to verify the efficacy of neuroprotective treatments. ${ }^{20,21}$ The hypothesis that neuronal death caused by ischemia involves an excitotoxic mechanism is not in opposition with the findings that the neuroprotective effect of neurotransmitter receptor agonists does not correlate with the reduction of glutamate release. ${ }^{22,23}$ In fact, it is possible that although some of the agents do not directly affect the glutamate release, they may modulate some events secondarily induced by glutamate receptor stimulation at the postsynaptic level, such as the activation of voltage-dependent inward conductances. It is also possible that some neuroprotective agents exert pharmacological effects during ischemia by modulating calcium-independent glutamate release. This neurotransmitter release pathology seems to be important during ischemia, and it may occur via different mechanisms. The protection exerted by phenytoin on ganglion cells and their axons (where ganglion cells compose $34 \%$ of the total macular volume) is reliable evidence of these mechanisms. Furthermore, some microglial responses are mediated via $\mathrm{Na}+$ channel activity. Therefore, the blockade of sodium channels could result in neuroprotective effects on the immune and nonimmune molecular pathways that may drive progressive nerve loss in MS. ${ }^{24}$ The partial inhibition of voltage-gated sodium channels during an episode of optic nerve inflammatory demyelination also prompted us to consider the protective roles of this compound. However, the beneficial effects of treatment on structural outcomes did not correlate very well with visual outcomes or visual-evoked potentials (VEP), according to the study by Raftopoulos et al. ${ }^{14}$ It might be that phenytoin protects neurons and their axons, while the functionality of these cells is irreversible. Phase III trials would be warranted to determine whether significantly improved clinical outcomes can be achieved. The use of sodium channel inhibitors with greater potency and specificity than phenytoin, along with testing higher drug concentrations through a similar mechanism of action, could be of great interest in elucidating the potential clinical utility of these compounds. An earlier treatment window during relapse than that assessed in previous studies might be considered. On the other hand, treatment with phenytoin was generally well-tolerated, and it was not associated with abnormalities in blood counts or liver function. In the studies reported, no acute deterioration of vision that might be attributed to the conduction block from the inhibition of sodium currents was observed. Since it was previously supposed that rebound deterioration upon the withdrawal of treatment could potentially limit the use of drugs acting on this target in demyelinating disorders, it was also important that this condition was not reported in the most recent trials. It is conceivable that other sodiumchannel inhibitors could also be neuroprotective in patients with acute optic neuritis and in other types of relapse in MS. This form of neuroprotection should improve relapse recovery in general, because the pathophysiology of optic neuritis resembles that of other cases of relapse in MS patients. This might represent a new foundation for combination studies in which phenytoin could be added to currently available disease-modifying therapies. In complex disorders leading to retinopathy, retinoprotection might have a greater therapeutic effect if phenytoin is combined with other compounds 
that display a comparable effect, but that act via an entirely different mechanism (such as palmitoylethanolamide). ${ }^{25}$ Palmitoylethanolamide has been used as an adjunct for cytoprotection in many different disorders characterized by slow inflammation. This strategy could be a means to further improve the effect of anti-inflammatory therapies on the preservation of retinal nerve cells and long-term outcomes for treating relapses in patients affected by demyelinating pathologies, such as MS.

\section{Disclosure}

The authors report no conflicts of interest in this work.

\section{References}

1. Willmore LJ. Antiepileptic drugs and neuroprotection: current status and future roles. Epilepsy Behav. 2005;7(Suppl. 3):S25-S28.

2. Grimm C, Hermann DM, Bogdanova A, et al. Neuroprotection by hypoxic preconditioning: HIF-1 and erythropoietin protect from retinal degeneration. Semin Cell Dev Biol. 2005;16(4-5):531-538.

3. Farkas RH, Grosskreutz CL. Apoptosis, neuroprotection, and retinal ganglion cell death: an overview. Int Ophthalmol Clin. 2001;41(1): 111-130.

4. Silverstein FS, Buchanan K, Hudson C, Johnston MV. Flunarizine limits hypoxia-ischemia induced morphologic injury in immature rat brain. Stroke. 1986;17(3):477-482.

5. Gill R, Foster AC, Woodruff GN. Systemic administration of MK-801 protects against ischemia-induced hippocampal neurodegeneration in the gerbil. J Neurosci. 1987;7(10):3343-3349.

6. Keltner JL, Becker B, Gay AJ, Podos SM. Effect of diphenylhydantoin in ischemic optic neuritis. Trans Am Ophthalmol Soc. 1972;70:113.

7. Ellenberger C, Burde RM, Keltner JL. Acute optic neuropathy. Treatment with diphenylhydantoin. Arch Ophthalmol. 1974;91(6):435-438.

8. Honda Y, Podos SM, Becker B. The effect of diphenylhydantoin on the electroretinogram of rabbits. I. Effect of concentration. Invest Ophthalmol. 1973;12(8):567-572.

9. Fern R, Ransom BR, Stys PK, Waxman SG. Pharmacological protection of CNS white matter during anoxia: actions of phenytoin, carbamazepine and diazepam. J Pharmacol Exp Ther. 1993;266(3):1549-1555.

10. Calabresi P, Picconi B, Saulle E, Centonze D, Hainsworth AH, Bernardi G. Is pharmacological neuroprotection dependent on reduced glutamate release? Stroke. 2000;31(3):766-773.
11. Lo AC, Black JA, Waxman SG. Neuroprotection of axons with phenytoin in experimental allergic encephalomyelitis. Neuroreport. 2002;13(15):1909-1912.

12. Thakral A, Shenoy R, Deleu D. Acute visual dysfunction following phenytoin-induced toxicity. Acta Neurol Belg. 2003;103(4):218-220.

13. Keppel Hesselink JM, Kopsky DJ. Phenytoin: neuroprotection or neurotoxicity? Neurol Sci. 2017;38(6):1137-1141.

14. Raftopoulos R, Hickman SJ, Toosy A, et al. Phenytoin for neuroprotection in patients with acute optic neuritis: a randomised, placebocontrolled, phase 2 trial. Lancet Neurol. 2016;15(3):259-269.

15. Miglior S, Zeyen T, Pfeiffer N, Cunha-Vaz J, Torri V, Adamsons I; European Glaucoma Prevention Study (EGPS) Group. Results of the European Glaucoma Prevention Study. Ophthalmology. 2005; 112(3):366-375.

16. Becker B, Stamper RL, Asseff C, Podos SM. Effect of diphenylhydantoin on glaucomatous field loss: a preliminary report. Trans Am Acad Ophthalmol Otolaryngol. 1972;76(2):412-422.

17. Naskar R, Quinto K, Romann I, Schuettauf F, Zurakowski D. Phenytoin blocks retinal ganglion cell death after partial optic nerve crush. Exp Eye Res. 2002;74(6):747-752.

18. Hains BC, Waxman SG. Neuroprotection by sodium channel blockade with phenytoin in an experimental model of glaucoma. Invest Ophthalmol Vis Sci. 2005;46(11):4164-4169.

19. McLean MJ, MacDonald RL. Multiple actions of phenytoin on mouse spinal cord neurons in cell culture. J Pharmacol Exp Ther. 1983; 227(3):779-789.

20. Lidster K, Baker D. Optical coherence tomography detection of neurodegeneration in multiple sclerosis. CNS Neurol Disord Drug Targets. 2012;11(5):518-527.

21. Raftopoulos RE, Kapoor R. Neuroprotection for acute optic neuritis can it work? Mult Scler Relat Disord. 2013;2(4):307-311.

22. Greene JG, Greenamyre JT. Bioenergetics and glutamate excitotoxicity. Prog Neurobiol. 1996;48(6):613-634.

23. Obrenovitch TP, Urenjak J. Altered glutamatergic transmission in neurological disorders: From high extracellular glutamate to excessive synaptic efficacy. Prog Neurobiol. 1997;51(1):39-87.

24. Morsali D, Bechtold D, Lee W, et al. Safinamide and flecainide protect axons and reduce microglial activation in models of multiple sclerosis Brain. 2013;136(Pt 4):1067-1082.

25. Keppel Hesselink JM, Costagliola C, Fakhry J, Kopsky DJ. Palmitoylethanolamide, a natural retinoprotectant: its putative relevance for the treatment of glaucoma and diabetic retinopathy. J Ophthalmol. 2015;2015:430596-430599.

\section{Publish your work in this journal}

Drug Design, Development and Therapy is an international, peerreviewed open-access journal that spans the spectrum of drug design and development through to clinical applications. Clinical outcomes, patient safety, and programs for the development and effective, safe, and sustained use of medicines are the features of the journal, which

\section{Dovepress}

has also been accepted for indexing on PubMed Central. The manuscript management system is completely online and includes a very quick and fair peer-review system, which is all easy to use. Visit http://www.dovepress.com/testimonials.php to read real quotes from published authors. 